\title{
Und wenn ich der Patient wäre?
}

\section{Hans Stalder}

Prof. Dr. med., Redaktionsmitglied

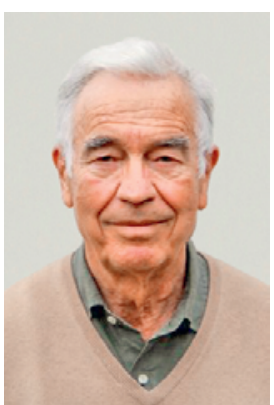

Die Literatur findet sich unter www.saez.ch $\rightarrow$ Aktuelle Ausgabe oder $\rightarrow$ Archiv $\rightarrow 2019 \rightarrow 22$
Siehe den Artikel «Medikamentöse Sekundärpräven tion nach Myokardinfarkt von Carola Huber und Thomas Rosemann auf Seite 779-781 dieser Ausgabe.
In dieser Ausgabe resümieren Carola Huber und Thomas Rosemann [1] einen Artikel, den sie im Januar in der Fachzeitschrift Clinical Therapeutics [2] veröffentlicht hatten und der sich mit der medikamentösen Adhärenz nach einem Herzinfarkt befasst. Der Artikel ist bemerkenswert und interessant. Bemerkenswert, da es sich um eine recht ungewöhnliche Zusammenarbeit zwischen einer Krankenkasse und einem Institut für Hausarztmedizin handelt, und interessant, weil die Autoren aufzeigen, dass nicht einmal 5\% der Patienten nach einem Infarkt regelmässig alle 5 empfohlenen Medikamente einnehmen - d.h. die duale Thrombozytenaggregationshemmung, einen Lipidsenker, einen Angiotensin-Converting-Enzymhemmer oder einen Angiotensin-Rezeptorblocker und einen Betablocker. Bei nur drei verschriebenen Medikamenten liegt die Anzahl jener, die den Vorgaben Folge leisten, bei etwa $7 \%$.

In einer jüngsten Zusammenfassung [3] wurden 80 Faktoren (!) identifiziert, die zur Nichtbeachtung von medikamentösen Guidelines beitragen, darunter - wie von Huber und Rosemann erwähnt - wie folgt die wahrscheinlichsten:

Zunächst einmal haben die Ärzte aufgrund von Kontraindikationen bei weitem nicht alle empfohlenen Medikamente verordnet; so erhält beispielsweise ein Patient, der wegen Vorhofflimmern Gerinnungshemmer einnimmt, keine Thrombozytenaggregationshemmer und ein Asthmatiker keine Betablocker. Es ist ausserdem anzunehmen, dass die Patienten das eine oder andere Medikament absetzen, da es zu Nebenwirkungen kam. Die Bereitschaft zur Einnahme von Betablockern (ca. 12\%) war besonders schwach. Vielleicht verlief die Kommunikation zwischen Arzt und Patient oder die Übermittlung der Daten zwischen Spital und Praxis nicht optimal. Ganz offenkundig ist das Problem der Polypharmazie. Es ist bekannt, dass die medikamentöse Adhärenz mit der Anzahl der verschriebenen Medikamente abnimmt [3]. Die meisten Patienten mit einem Altersdurchschnitt von 68,5 Jahren (wie in der Studie) mussten nicht nur neue Medikamente einnehmen, sondern nahmen schon etliche vor dem Infarkt [4].

Meines Erachtens kommt noch ein weiterer Faktor dazu: finanzielle Probleme. Eine Kostenbeteiligung bei den Medikamenten ist besonders problematisch für
Menschen mit multiplen Pathologien, da sie deswegen oft ganz auf die Behandlung verzichten. Es wurde nachgewiesen, dass ein Aussetzen der Kostenbeteiligung nach einem Infarkt das Überleben der Patienten und ihre Lebensqualität verbessert, ohne die Gesamtkosten zu erhöhen [5, 6]. (Ganz offenkundig lassen solche Daten unsere Parlamentarier, die den Selbstbehalt noch erhöhen wollen, kalt.)

Die Einnahme von Medikamenten war mit einer besseren Prognose assoziiert. Gemäss den Autoren ist es daher wahrscheinlich, dass eine bessere Einhaltung der Vorgaben für bessere Überlebenschancen sorgt oder zumindest weitere kardiovaskuläre Vorfälle einschränkt. Allerdings waren das fortgeschrittene Alter und die Komorbiditäten noch stärker mit negativen Überlebenschancen verknüpft. Das Sprichwort «besser jung, reich und gesund" gilt auch nach einem Infarkt .. Diese Arbeit zeigt die Grenzen der Anwendung der Evidence-based Medicine beim individuellen Patienten auf. Einzeln betrachtet wurde die Wirksamkeit jedes in den Richtlinien vorgeschlagenen Medikaments bewiesen - jedenfalls im Vergleich zum Placebo und im Allgemeinen bei Patienten ohne Komorbiditäten. Aber was nützt das, wenn die Ärzte die Medikamente nicht verschreiben und die Patienten sie nicht nehmen? Es gibt keine einfache Lösung für diese Problematik! Versetzen Sie sich für einen Moment in die Person des Patienten. Würden Sie nach einem Infarkt zusätzlich zu zwei oder drei bereits verordneten Medikamenten noch 5 weitere einnehmen? Zumal alle präventiv verschrieben werden; Sie haben ja keine Symptome mehr. Verschlimmert sich die Prognose, wenn ich das eine oder andere Medikament weglasse? Soll ich das Antidepressivum, das mir so geholfen hat, absetzen - oder das Antidiabetikum, das Antiasthmatikum oder die Vitamine? Kann ich mein Schmerzmittel, ohne das ich nicht gut gehen kann, weiter einnehmen?

Wie die Autoren können wir die Antworten nur vermuten. Um sie wirklich zu kennen, müssten wir jeden Patienten einzeln nach dem Warum fragen. Dies könnte im Rahmen einer neuen, dieses Mal wohl eher qualitativen Studie geschehen - und warum nicht in Zusammenarbeit einer Krankenkasse mit Ärzten: «Warum nehmen Sie die Medikamente, die Ihnen der Arzt verschrieben hat, nicht ein?» 
Literatur

1 Huber CA, Rosemann T. Medikamentöse Sekundärprävention nach Myo kardinfarkt. Schweiz Ärzteztg. 2019;100(22):779-81.

2 Huber CA, Meyer MR, Steffel J, Blozik E, Reich O, Rosemann T. Post-myocardial infarction (MI) care: medication adherence for secondary prevention after MI in a large real-world population. Clin Ther. 2019;41:107-17.

3 Yap AF, Thirumoorthy T, Kwan YH. Systematic review of the barriers affecting medication adherence in older adults. Geriatr Gerontol Int. 2016;16: 1093-101.

4 https://www.bfs.admin.ch/bfs/de/home/aktuell/neue-veroeffentlichungen.gnpdetail.2019-0117.html

5 Ito K, Avorn J, Shrank WH, Toscano M, Spettel C, Brennan T, Choudhry NK Long-term cost-effectiveness of providing full coverage for preventive medications after myocardial infarction. Circ Cardiovasc Qual Outcomes. 2015;8:252-9.

6 Choudhry NK, Patrick AR, Antman EM, Avorn J, Shrank WH. Cost-effectiveness of providing full drug coverage to increase medication adherence in post-myocardial infarction Medicare beneficiaries. Circulation. 2008;117:1261-8. 